\title{
Development and Validation of Stability-Indicating Ultra High-Performance Liquid Chromatography for Ramipril Analysis in Pharmaceutical Dosage Forms and Its Application in Lipid-based Formulations
}

\author{
KHALID FAISAL ALHASANI' ${ }^{1}$ KAZI MOHSIN ${ }^{\star 1}$, FAIYAZSHAKEEL $^{1,2}$ and FARS KAED ALANAZI ${ }^{1}$ \\ ${ }^{1}$ Kayyali Chair for Pharmaceutical Industries, College of Pharmacy, King Saud University, Riyadh, \\ Saudi Arabia. \\ ${ }^{2}$ Center of Excellence in Biotechnology Research, King Saud University, Riyadh, Saudi Arabia. \\ ${ }^{*}$ Corresponding author E-mail: mkazi@ksu.edu.sa
}

http://dx.doi.org/10.13005/ojc/340107

(Received: August 26, 2017; Accepted: September 11, 2017)

\begin{abstract}
The current study evaluates the Ultra High performance Liquid Chromatography (UHPLC) method for the quantification of Ramipril in lipid-based formulations. A reliable, highly precise, more specific and reproducible reversed phase UHPLC method has been developed and validated according to the regulatory guidelines, which was composed of isocratic mobile phase; acetonitrile and $0.25 \%$ formic acid solution in ratio of $(40: 60 \% \mathrm{~V} / \mathrm{V})$ with a flow rate of $0.2 \mathrm{~mL} / \mathrm{min}$, and $\mathrm{C} 18$ column $(2.1 \times 50 \mathrm{~mm}, 1.7 \mu \mathrm{m})$. The detection was carried out at $210 \mathrm{~nm}$. The developed UHPLC method was found to be rapid ( 3 min. run time), selective with high resolution of Ramipril peak (1.4 min.) from different lipid matrices and highly sensitive (Limit of Detection and Lower Limit of Quantification were $0.034 \mu \mathrm{g} / \mathrm{mL}$ and $0.199 \mu \mathrm{g} / \mathrm{mL}$, respectively). The linearity, accuracy and precision were determined as acceptable over the concentration range of $1-200 \mu \mathrm{g} / \mathrm{mL}$ for Ramipril. The results showed that the proposed UHPLC method can be used for the estimation of Ramipril in lipid-based formulation by indicating its purity and stability with no interference of excipients or related substances of active pharmaceutical ingredient.
\end{abstract}

Keywords: Ramipril, UHPLC systems, Lipid based formulation, Method validation.

\section{INTRODUCTION}

Ramipril (RMP) is (Fig.1) chemically named as 2-[N-[(S)-1-ethoxycarbonyl-3-phenylpropyl] - L - alanyl] - (1S, 3S, 5S) - 2 - azabicyclo[3,3,0]- octane-3-carboxylic acid ${ }^{1}$. It is classified as angiotensin converting enzyme inhibitor (ACE) ${ }^{2,3}$. It is mainly used for the treatment of hypertension and heart failure ${ }^{4}$. The doses of RMP for these indications range is between $2.5-20 \mathrm{mg}$ daily as

This is an 2 Open Access article licensed under a Creative Commons Attribution-NonCommercial-ShareAlike 4.0 International License (https://creativecommons.org/licenses/by-nc-sa/4.0/ ), which permits unrestricted NonCommercial use, distribution and reproduction in any medium, provided the original work is properly cited. 
the patients require well tolerated low doses ${ }^{5}$. RMP is orally active but not giving the pharmaceutical activity unless goes after metabolism by the liver to produce its active moiety, ramiprilate $e^{6,7}$

RMP is classified as Class II in Biopharmaceutical Classification System (BCS) which is presented by Amidon in 1995. RMP is considered as poorly bio-available because it has low water solubility but highly permeable through GIT barriers which gives a bioavailability of $28.35 \%$. Partition coefficient $(\log p)$ of RMP is 3.41 and its pka is 3.17 . RMP is a highly challenging compound which poses stability problems in acidic and alkaline media and it is sensitive to heat, moisture and mechanical stress ${ }^{8-10}$.

Many studies have been published previously to analyze RMP using conventional method in pharmaceutical dosage forms. In 2005, Nafisur Rahman et al., published a kinetic spectrophotometric analysis method using color reaction. The method was based on the reaction of carboxylic group of RMP with a mixture of iodideiodate in aqueous medium resulting in the formation of yellow color, which absorbs maximally at 352 nm. In 2007, Hisham Abdullatef investigated the detection of RMP by different spectrophotometric and spectrofluorometric methods by oxidation of RMP with 1-chlorobenzotriazole (CBT) to be measurable at absorbance of $350 \mathrm{~nm}^{11}$. Another method was done by formation of ternary complex of Copper II, eosin and RMP with surfactant to be detected at absorbance of $543 \mathrm{~nm}$. These methods were able to detect a range of 20 to $80 \mu \mathrm{g} / \mathrm{mL}$. In 2010, M. De Diego et al., worked in liquid chromatography analysis combined with stress conditions analysis, succeeded to detect RMP as stability indicating method.

However, the procedures used for method development in most of the papers are laborious and time consuming and/or covering only narrow concertation ranges. The aim of this work is to develop and validate a rapid, simple, accurate, precise, economic, stability indicating and wide concentration detectable range method to detect RMP in pharmaceutical dosage forms with the application of lipid based formulation.

\section{EXPERIMENTAL}

\section{Materials}

Ramipril was obtained as a kind gift sample from Zydus Cadila Healthcare Ltd. (Ahmedabad, Gujrat, India). HPLC grade Acetonitrile, hydrochloric acid and Formic acid were purchased from BDH laboratory supplies $(\mathrm{BDH}$ Chemicals Ltd., Poole, UK). orthophosphoric acid has been purchased from (Avonchem, Cheshire, UK). Oils used are Miglyol 812 (M812: Medium chain triglycerides (72\% C8 and $27.5 \% \mathrm{C} 10)$ and Imwitor 988 (I988 Caprylic / Capric Glycerides) are supplied by (Sasol GmbH, Witten, Germany and Cremer Oleo GmbH \& Co. KG. Hamburg, Germany) respectively. The high purity Milli-Q water was obtained through a Milli-Q Integral Water Purification System (Millipore, Bedford, MA). Ramipril $2.5 \mathrm{mg}$ tablet Sandos $₫$ was used as marketed drug. Hydrogen peroxide solution was obtained from (S.G.H Harborough, England) and sodium hydroxide from (Merck, Germany), respectively. All other reagents were of analytical grade and used without further purification.

\section{METHODS}

\section{UHPLC chromatographic studies}

Development and optimization of chromatographic separation was done with respect to the compositions of mobile and stationary phases, column temperature, flow-rate, sample volume, and detection wavelength. The work within the scope used a highly sensitive UHPLC system that composed of a Dionex $₫$ UHPLC binary solvent manager equipped with a Dionex ${ }^{\circledR}$ automatic sample manager and a Photodiode Array (PDA) e $\lambda$ detector by Thermo scientific, Bedford, MA, USA. The mobile phase was an isocratic mixture of HPLCgrade acetonitrile and $0.25 \%$ formic acid aqueous solution in a ratio of $(40: 60 \% \mathrm{v} / \mathrm{v})$. The flow rate was $0.2 \mathrm{~mL} / \mathrm{min}$. connected through an Acquity® UPLC BEH C18 column $(2.1 \times 50 \mathrm{~mm}, 1.7 \mu \mathrm{m})$ with temperature of $50{ }^{\circ} \mathrm{C}$. The total run time was extended to $3 \mathrm{~min}$. whereas the RMP eluted at $1.4 \mathrm{~min}$. only. Freshly prepared mobile phase was filtered through an online $0.20 \mu \mathrm{m}$ filter and degassed continuously by an online degasser within the UHPLC system. The detector wavelength was set at $210 \mathrm{~nm}$ and the injection volume was 2.0 
$\mu \mathrm{l}$. The solvent for sample dilution was prepared by mixture of acetonitrile and $(3 \% \mathrm{v} / \mathrm{v}$ orthophosphoric aqueous solution) with a ratio of 2:3.

\section{Preparation of stock solution, calibration standards and QC samples}

Preparation of calibration standards, quality control (QC) samples and stock solution of RMP were prepared in the designated solvent (orthophosphoric acid \& ACN mix) at a concertation of $1 \mathrm{mg} / \mathrm{mL}$. The stock solutions were consecutively diluted with the solvent to prepare working solutions just before use. RMP standards were prepared by diluting of $125,250,625,1250,2500$ and $5000 \mu \mathrm{l}$ RMP working standard stock solution by $25 \mathrm{~mL}$ solvent in volumetric flask to obtain six-point calibration concentrations of $5-200 \mu \mathrm{g} / \mathrm{mL}$ (ppm). $10 \mu \mathrm{g} / \mathrm{mL}$ solution diluted by 10 times to produce lower concentration of $1 \mu \mathrm{g} / \mathrm{mL}$ This procedure made concentrations of 1, 5, 10, 25, 50, 100, 200 $\mu \mathrm{g} / \mathrm{mL}$ (ppm). Calibration curves were obtained by plotting peak area against standard drug concentration and regression equations were computed thereby. Four quality control (QC) samples with the selected concentrations of 18,36 , 78 and $140(\mu \mathrm{g} / \mathrm{mL})$ ppm were prepared to cover the desired range from the calibration standards. Three tablets from the marketed drug (Ramipril Sandos $® 2.5 \mathrm{mg}$ ) dissolved in $25 \mathrm{~mL}$ of solvent to assure the potentiality of the method that can be applied to assay RMP in commercial formulations.

\section{Method Validation}

The current developed method was validated according to $\mathrm{ICH}$ guidelines in terms of linearity, specificity, precision and accuracy, Limit of Detection (LOD) and Lower Limit of Quantification (LLOQ) according to the standard guidelines of bioanalytical method validation ${ }^{12}$. The acceptance criteria of precision and accuracy were evaluated as the relative standard deviation of the mean expressed as a percent (coefficient of variation, CV\% determined precision of the method) ${ }^{13,14}$

\section{Linearity and range}

Appropriate volumes of RMP stock solutions $(1 \mathrm{mg} / \mathrm{mL})$ were utilized to prepare seven non-zero standard drug concentrations covering the calibration range of $1-200 \mu \mathrm{g} / \mathrm{mL}$. Four QC samples were prepared by spiking known concentrations of RMP within the detection range of $1-200 \mu \mathrm{g} / \mathrm{mL}$. Each standard solution 1, 5, 10, $25,50,100,200 \mu \mathrm{g} / \mathrm{mL}$ has been injected $(n=3)$ times daily on three consecutive days for validation. In ascending order, calibration solutions were injected in each validation run and the other samples were randomly distributed through the run. Correlation coefficient $\left(R^{2}\right)$ and linear regression equation were incorporated to statistically evaluate the linearity of the results, $y=m x+b$, where; $y=$ response. $\mathrm{m}=$ slope. $\mathrm{x}=$ concentration, $\mathrm{b}=$ intercept.

\section{Specificity}

Specificity and sensitivity of the method were evaluated in relation to observe any interference peaks from the different self-emulsifying lipid-based formulations and the peak of the standard solutions. The retention time for both peaks were compared to assure the same time is obtained. In addition, towards the establishment of the method, specificity was also studied by determination of the intact drug in terms of resolution $(R)$ between the drug peak and the nearest degradation product if present.

\section{Accuracy and precision}

From the seven RMP standard solutions, intra-day accuracy and precision studies were performed by using six replicates determinations within the same day. On the other hand, the interday accuracy and precision were also assessed by six replicates analysis during the three consecutive days of the following: low and high QC samples. The overall precision of the method was expressed as relative standard deviation (RSD).

\section{Limit of Detection (LOD) and Lower Limit of Quantification (LLOQ)}

The LOD and LLOQ were determined efficiently by serial dilutions of the RMP stock solution in order to obtain signal to noise $(\mathrm{S} / \mathrm{N})$ ratio of at least H" 3:1 for LOD and H" 10:1 for LLOQ.

\section{RESULTS AND DISCUSSION}

\section{UHPLC peak separation}

The representative chromatographic results of blank solvent $(A)$, drug free selfemulsifying lipid formulation sample (B), RMP standard solution $100 \mu \mathrm{g} / \mathrm{mL}$ (C), RMP containing self-emulsifying lipid formulation sample (D) and 
the marketed drug sample (E) are shown in Fig. 2. The chromatographic results found from the current UHPLC technique showed that the RMP peaks were clearly separated and ideal without any interference of the excipients used in liquid dosage form. In addition, the chromatograms in the assay confirmed that the sensitivity and selectivity of this procedure was efficient enough to determine RMP within the available excipients used for dosage forms. The RMP analyte was well separated at the retention time of $\sim 1.4 \mathrm{~min}$. without having any interference of degradation product. The total run time was $\sim 3$ min., where the peaks were of good shape and completely resolved.

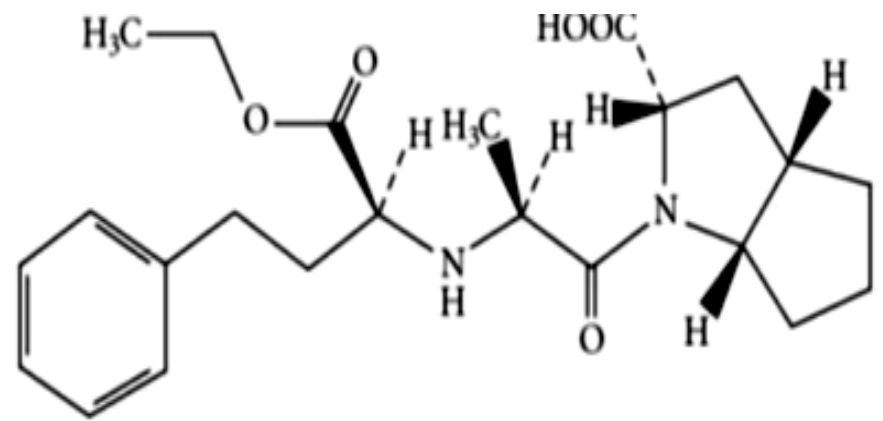

Fig. 1. Chemical structure of RMP (MW: 416.511, PKa: 3.17).
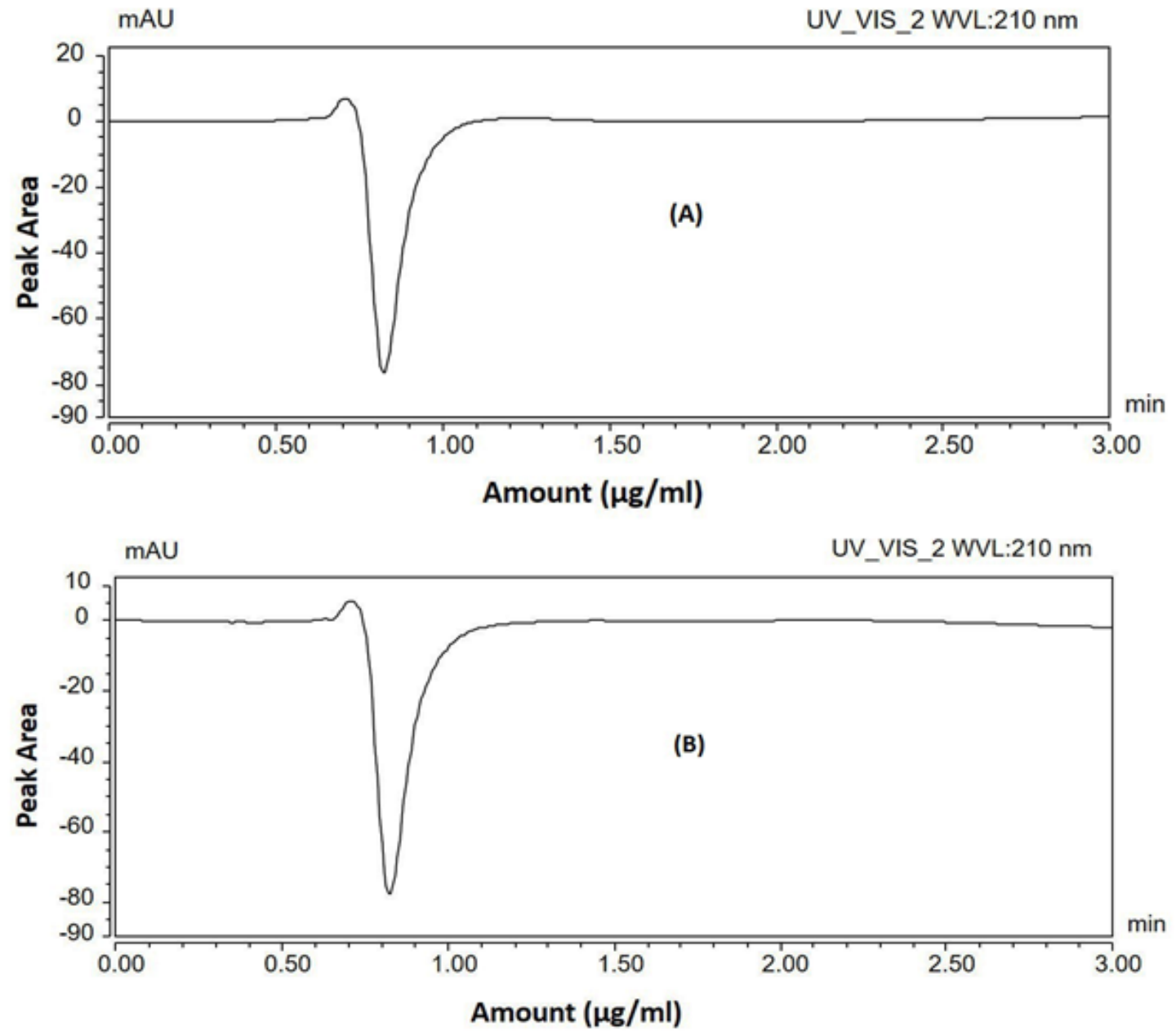

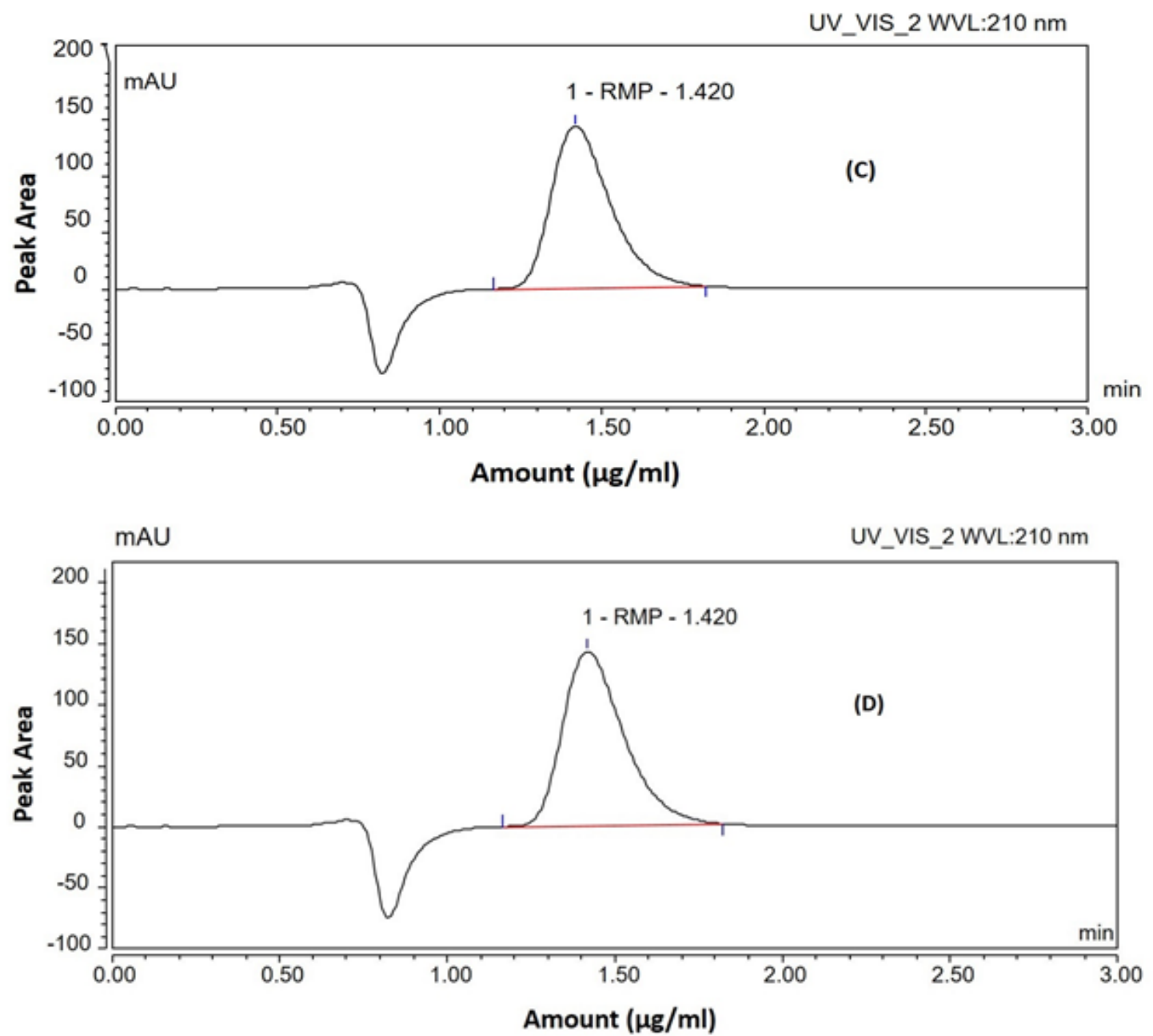

UV_VIS_2 WVL:210 nm

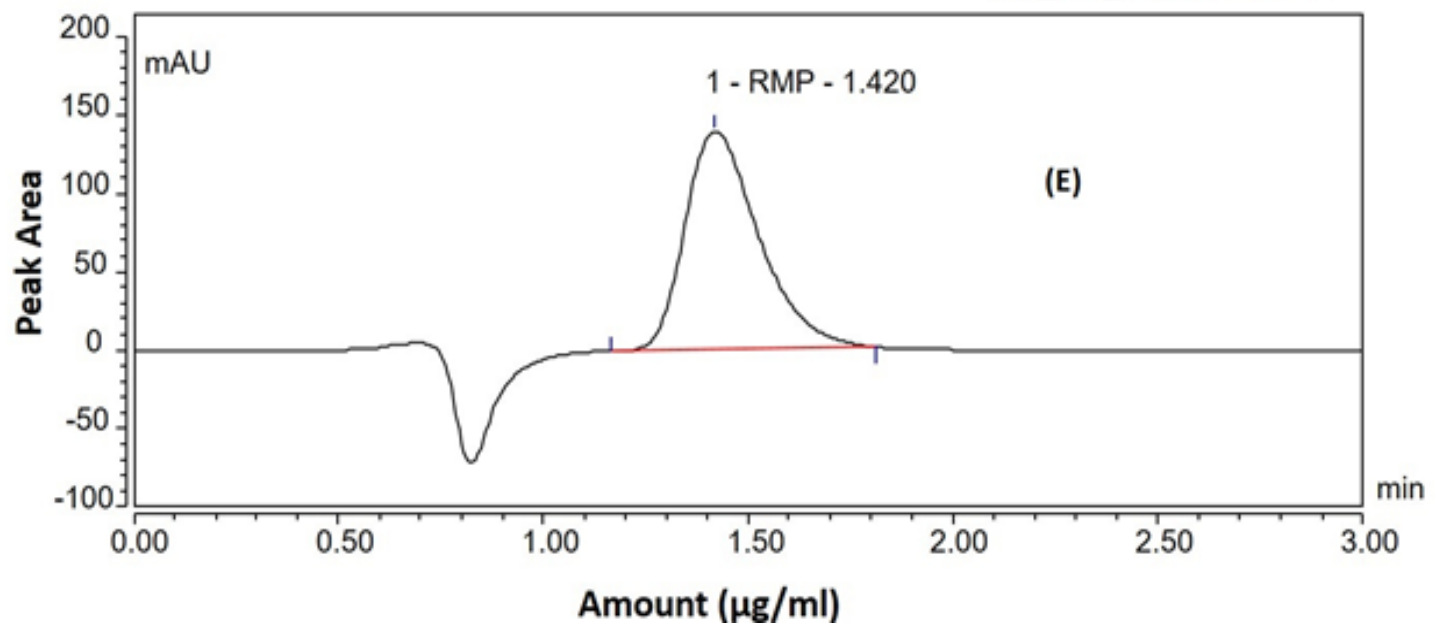

Fig. 2. UHPLC chromatograms of blank sample (A), drug-free lipid formulation sample (B), standard solution of RMP at concentration $100 \mathrm{ppm}$ (C), and drug-containing lipid formulation sample (D). RMP Sandos $\circledast 2.5 \mathrm{mg}(\mathrm{E})$ 


\section{Forced degradation study}

To study the ability of this method to be used as stability indicating method, five different samples of RMP was treated with stress conditions of $0.1 \mathrm{~N}$ hydrochloric acid, $0.1 \mathrm{~N}$ sodium hydroxide solution, $3 \%$ hydrogen peroxide solution and exposed to high temperature. The results showed by the degradation study that the amounts of RMP in the samples were decreased in compare to the original concentrations.

\section{Acid degradation}

$10 \mathrm{mg}$ of RMP was transferred into a $100 \mathrm{~mL}$ round bottom flask and then $10 \mathrm{~mL}$ of $1 \mathrm{M}$ hydrochloric acid solution was added. The contents of the flask were mixed well and kept for overnight at $60{ }^{\circ} \mathrm{C}$ temperature. After cooling the solution at room temperature, $10 \mathrm{~mL}$ of $1 \mathrm{M}$ sodium hydroxide solution was added in $100 \mathrm{~mL}$ volumetric flask to neutralize and then diluted to $100 \mathrm{ML}$ with solvent. The chromatogram of the acid degradation is shown in Fig. 3A.

UV_VIS_2 WVL:210 nm

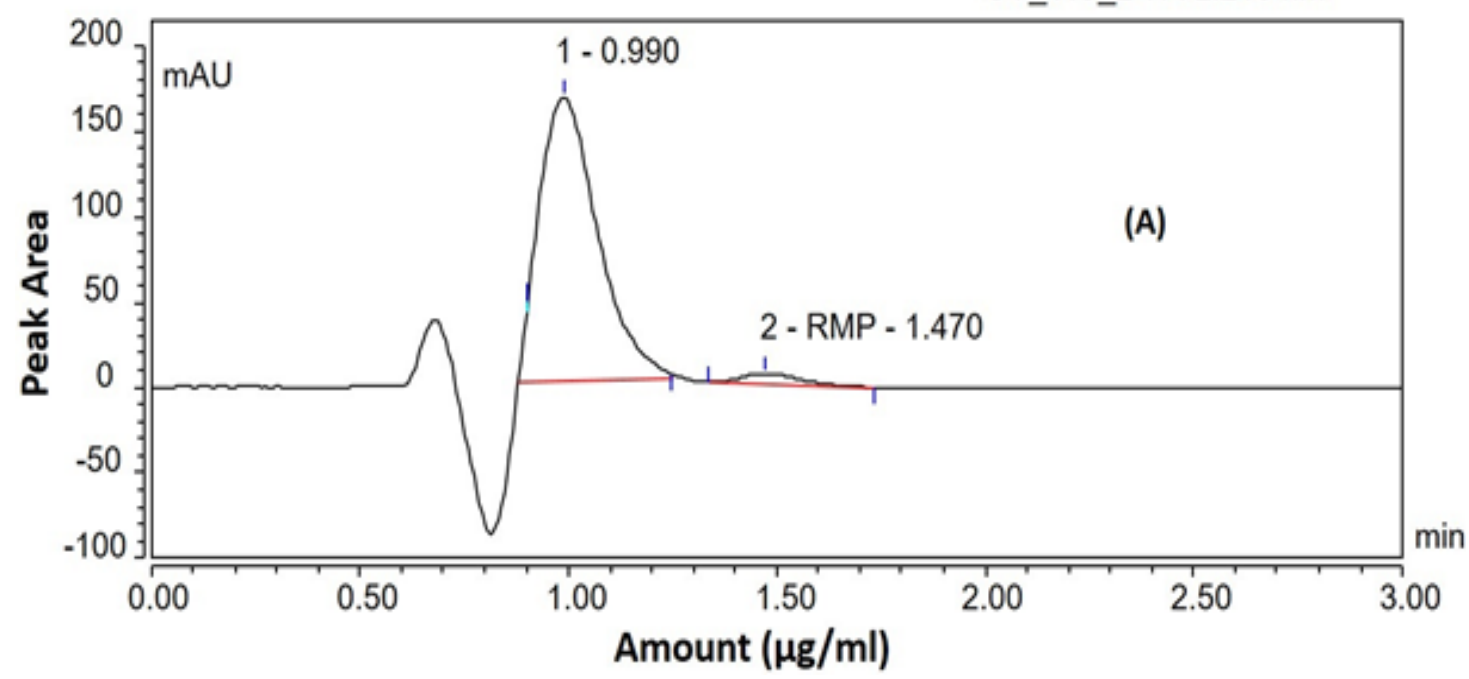

UV_VIS_2 WVL:210 nm

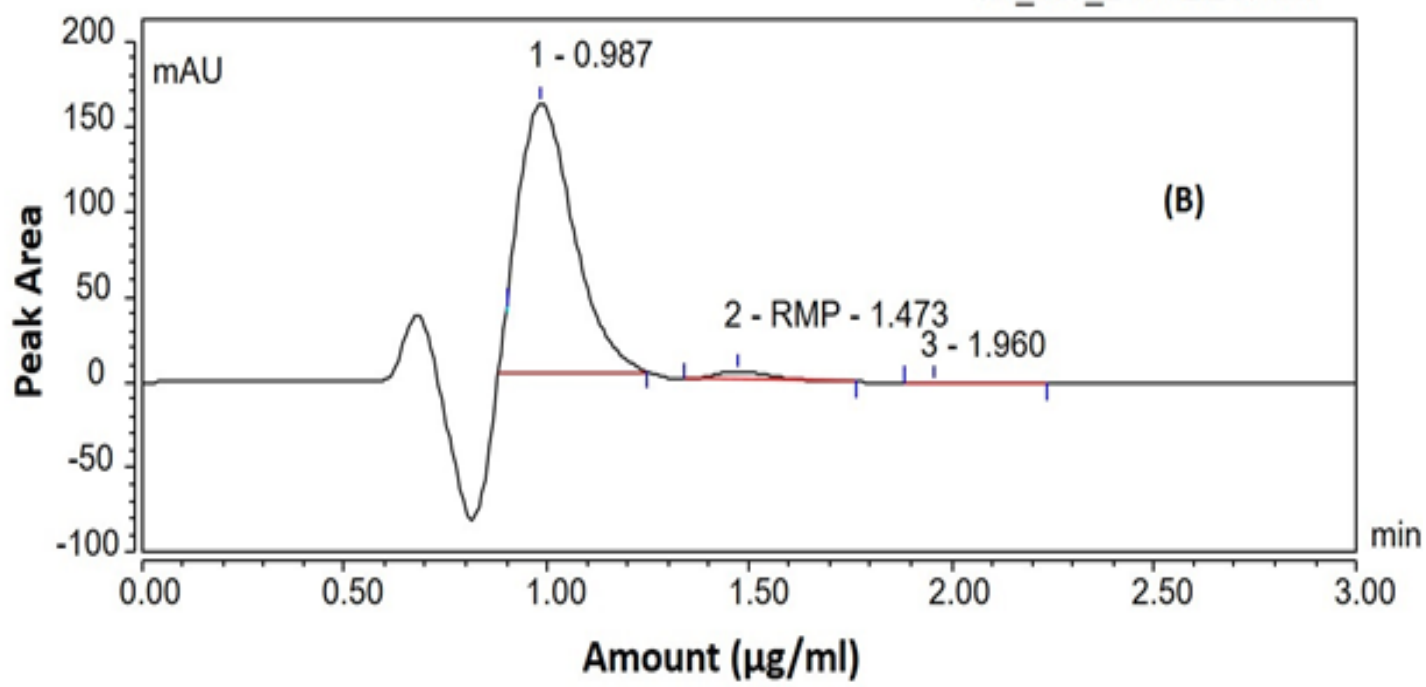


UV_VIS_2 WVL:210 nm
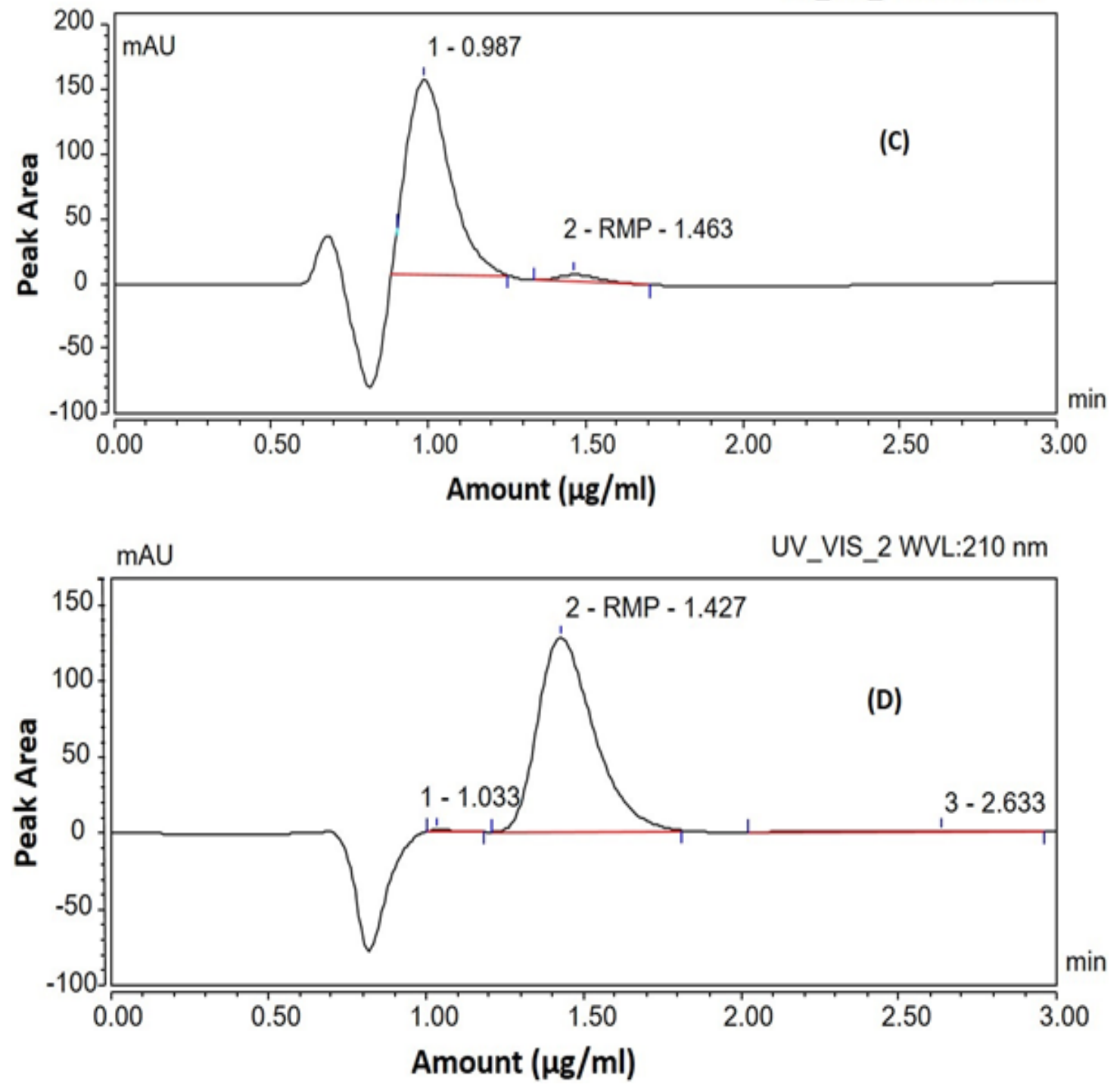

Fig 3. Typical UHPLC chromatograms of: (A) acid hydrolysis-degraded RMP, (B) base hydrolysisdegraded RMP, (C) oxidative-degraded RMP, and (D) thermal-degraded RMP.

\section{Basic degradation}

$10 \mathrm{mg}$ of RMP was transferred into a $100 \mathrm{~mL}$ round bottom flask and then $10 \mathrm{~mL}$ of $1 \mathrm{M}$ sodium hydroxide solution was added. The contents of the flask were mixed well and kept for overnight at $60^{\circ} \mathrm{C}$ temperature. After cooling the solution at room temperature, $10 \mathrm{~mL}$ of $1 \mathrm{~m}$ hydrochloric acid solution was added in $100 \mathrm{~mL}$ volumetric flask to neutralize and then diluted to $100 \mathrm{~mL}$ with solvent. The result of the basic degradation is shown in Fig. 3B.

\section{Oxidation}

$10 \mathrm{mg}$ of RMP was transferred into a $100 \mathrm{~mL}$ round bottom flask with the addition of $10 \mathrm{~mL} \mathrm{3 \%} \mathrm{hydrogen} \mathrm{peroxide} \mathrm{solution.} \mathrm{The}$ contents of the flask were mixed well and kept for overnight at $60^{\circ} \mathrm{C}$ temperature. After cooling the solution at room temperature, it was diluted to 100 $\mathrm{mL}$ with solvent. The chromatogram of the oxidation data is shown in Fig. $3 \mathrm{C}$.

\section{Thermal degradation}

$10 \mathrm{mg}$ of RMP was transferred into a 
$100 \mathrm{~mL}$ round bottom flask, and kept in oven at $120{ }^{\circ} \mathrm{C}$ for 24 hours. After cooling the powder at room temperature, the solvent was added up to 100 $\mathrm{mL}$. The chromatogram of the thermal degradation study is shown in Fig. 3D.

\section{Method validation}

Linearity, range and calibration

The peak responses of RMP were linear over the concentration range between 1 and 200 $\mu \mathrm{g} / \mathrm{mL}$. Under the above described experimental conditions, the calibration curve of chromatographic peak area versus RMP concentrations have been shown to be well linear dynamic range (Fig. 4). These results showed a perfect linear method over the interval studied with correlation coefficient, RMP $\left(r^{2}\right)=.99999$. Slope, intercept, correlation coefficient (r2), standard deviation of slope and intercept (obtained by the linear least squares treatment of the results) are listed in Table. 3. The LOD and LOQ of the method were found to be 0.034 and 0.199 respectively.

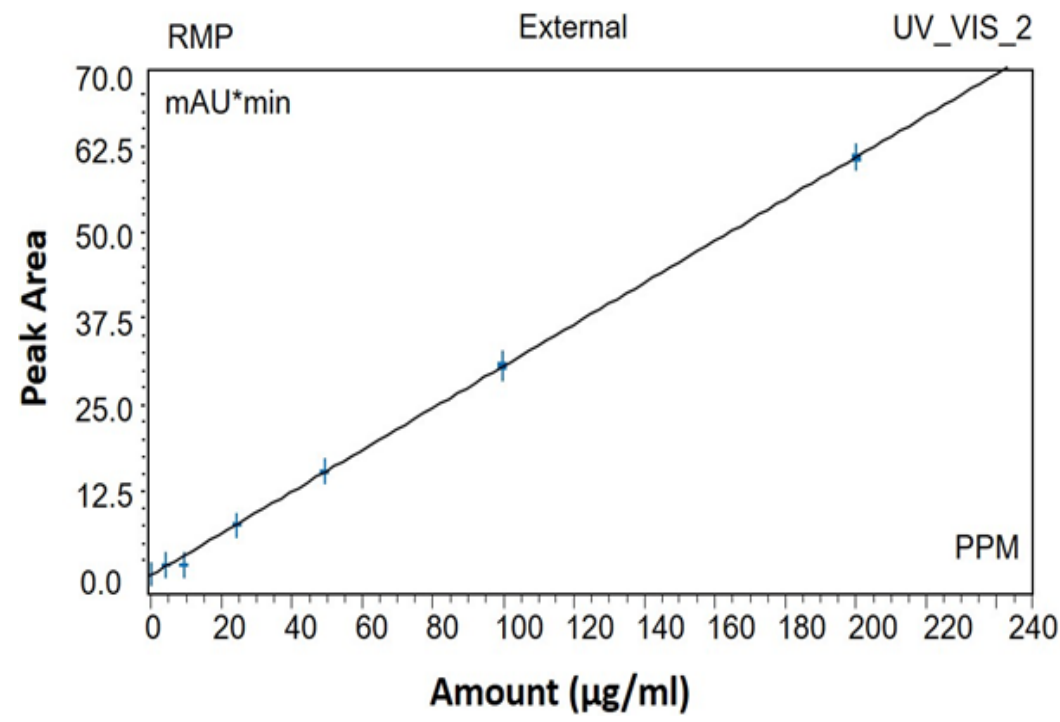

Fig. 4. UHPLC calibration curve of RMP in Solvent

Table. 1: UHPLC data of intra-day back-calculated RMP concentrations of the calibration standards in Solvent

\begin{tabular}{lcccc}
\hline Assay Type & Added & $\begin{array}{c}\text { Amount }(\mu \mathrm{g} / \mathrm{mL}) \\
\text { Found } \pm \mathrm{SD}\end{array}$ & Accuracy $(\%)$ & Precision \\
\hline Inter day & 1 & $1.067 \pm 0.013$ & 106.667 & \\
& 5 & $5.046 \pm 0.005$ & 100.913 & 0.093 \\
& 10 & $10.012 \pm 0.015$ & 100.122 & 0.152 \\
& 25 & $24.991 \pm 0.003$ & 99.965 & 0.01 \\
& 50 & $49.786 \pm 0.071$ & 99.573 & 0.143 \\
& 100 & $99.802 \pm 0.102$ & 99.802 & 0.102 \\
& 200 & $200.257 \pm 0.049$ & 100.128 & 0.024 \\
& 1 & $0.971 \pm 0.002$ & 97.05 & 0.218 \\
& 5 & $4.987 \pm 0.004$ & 99.73 & 0.081 \\
& 10 & $9.927 \pm 0.002$ & 99.273 & 0.025 \\
& 25 & $24.965 \pm 0.003$ & 99.859 & 0.010 \\
& 50 & $49.826 \pm 0.078$ & 99.652 & 0.157 \\
& 100 & $99.964 \pm 0.031$ & 99.964 & 0.031 \\
& 200 & $200.093 \pm 0.045$ & 100.047 & 0.023 \\
\hline
\end{tabular}


Table. 2: UHPLC data of inter-day accuracy and precision of RMP QC samples

\begin{tabular}{lcccc}
\hline Assay Type & \multicolumn{2}{c}{ Amount $(\mu \mathrm{g} / \mathrm{mL})$} & Accuracy $(\%)$ & Precision \\
& 18 & Found \pm SD & & \\
\hline \multirow{2}{*}{ QC Samples } & 36 & $18.332 \pm 0.015$ & 101.842 & 0.084 \\
& 78 & $36.082 \pm 0.016$ & 100.227 & 0.044 \\
& 140 & $78.010 \pm 0.005$ & 100.012 & 0.007 \\
& $2.5 \mathrm{mg} / 25 \mathrm{~mL}$ & $141.715 \pm 0.878$ & 101.225 & 0.62 \\
Marketed Drug & $99.683 \pm 0.200$ & 99.683 & 0.2 \\
(Sandos $\left.{ }^{2} 2.5 \mathrm{mg}\right)$ & solvent $(100 \mathrm{ppm})$ & & & \\
\hline
\end{tabular}

Table. 3: Statistical data of the regression equation for the determination of RMP obtained from the proposed method

\begin{tabular}{lc}
\hline Parameters & RMP \\
\hline Concentration range $(\mu \mathrm{g} / \mathrm{mL})$ & $1-200$ \\
Intercept $(\mathrm{a})$ & 0.0464 \\
Slope $(\mathrm{b})$ & 1.0005 \\
Correlation coefficient $(\mathrm{r})$ & 0.99999 \\
Limit of detection $(\mathrm{LOD})$ & 0.034 \\
Lower limit of quantification (LLOQ) & 0.199 \\
\hline
\end{tabular}

amean of ten measurements

'Limit of detection was estimated at a signal-to-noise ratio of 3

'Limit of quantification was estimated at a signal-to-noise ratio of 10

Table. 4: Systems suitability parameters of RMP

\begin{tabular}{lc}
\hline System suitability parameter & RMP \\
\hline Retention Time & $1.400 \mathrm{~min}$. \\
$\%$ RSD & $0.054 \%$ \\
Peak Tailing & 1.05 \\
Theoretical Plate number & 2829 \\
\hline
\end{tabular}

\section{Accuracy and precision}

The intra-day and inter-day accuracy studies was calculated as the percentage of drug recovery after examining six replicates of $Q C$ samples at four nominal concentration levels $(18,36,78$ and $140 \mu \mathrm{g} / \mathrm{mL}$ ) along with the seven standards. The data shows that the intra-day accuracy was found in between $97.05 \%$ and $100.541 \%$ and the inter-day accuracy was in between $99.573 \%$ and $100.666 \%$ (Table. 1, 2). The assay of the marketed drug was ideal with presented method as the accuracy was $99.683 \%$ of the label content. The overall results from the drug recovery suggest the accuracy of the assay method, which meet all the criteria as per the $\mathrm{ICH}$ guidelines.

The values present in (Table. 1, 2) Shows the results of intra-day and inter-day precision, respectively. The standard deviation (SD) values from the intra-day results are within the acceptable range, which indicates that the developed method was precise. In addition, within the analytical concentration range of $1-200 \mu \mathrm{g} / \mathrm{mL}$, the coefficient of variation ( $\mathrm{CV}$; precision) was found to be within 0.010 to $1.262 \%$. The inter-day (Table. 1,2$)$. SD values of six replicate determinations in three consecutive days were within the acceptable range, whereas the \% CV was in between the range of $0.024 \%$ and $1.262 \%$. These low values of both SD and $\mathrm{CV}$ during the intra-day and inter-day analysis suggest the current method as very highly precise.

\section{Specificity}

The specificity of the developed UHPLC method was investigated in order to measure the 
analyte response in the presence of its degradation products. The developed method was found to be specific for RMP without having any possible interference from the degradation product(s). The result from a sample of lipid solubility studies (RMP loaded in lipid formulation) in Fig. 2D shows that there was no degradation product present in the sample containing RMP. It seems that RMP compound can be recovered completely from the lipid formulation (Fig. 2D). In addition, there were no significant interfering peaks present in randomly selected drug free lipid formulation samples at RMP retention time (Fig. $2 \mathrm{~B}$ ), which suggests that RMP compound can be analyzed predominantly from lipid based formulations.

\section{Limit of Detection (LOD) and Lower Limit of Quantification (LLOQ)}

The LLOQ value of $0.199 \mu \mathrm{g} / \mathrm{mL}$ in the assay which was estimated to be the lowest concentration in the standard curve that can be measured with acceptable accuracy and precision for the RMP analyte with $\mathrm{S} / \mathrm{N}$ ratio of (79.7). On the other hand, LOD was $0.034 \mathrm{ppm}$ with $\mathrm{S} / \mathrm{N}$ ratio of (27.8). Both LLOQ and LOD were experimentally verified by six replicate injections of RMP standard concentrations (Table. 3).

\section{Suitability of the systems}

The system suitability was considered to estimate/settle the performance (parameters) and the highest precision of the systems. The variation (SD) in the peak area from six replicates injections was very minor, which demonstrates the system precision. The results of other chromatographic parameters such as peak tailing and theoretical plate numbers represents column efficiency are represented in Table. 4. The overall analysis of the results showed the acceptable/highest performance of the system as the tailing peaks was 1.05 along with the theoretical plates, which were not less than 2000.

\section{Application}

Within the scope of the current research, the developed UHPLC method has been successfully used for the quantification of RMP compound in the studies of equilibrium solubility, tablets assay and dissolution profiles of self-emulsifying lipid-based formulations (SEDDS/ SMEDDS).

\section{CONCLUSION}

The developed UHPLC method provides a reliable, highly precise and more specific assay for RMP analysis in pharmaceutical formulations. The described method is sensitive enough to detect as low as $0.034 \mu \mathrm{g} / \mathrm{mL}$ RMP and exclusively offer a rapid determination of RMP (peak at 1.4 min. within $3 \mathrm{~min}$. run time). No significant interferences were caused by the formulation excipients, diluents and or degradation products. The validation method allows quantification of RMP in pure and pharmaceutical formulations in the range between $1-200 \mu \mathrm{g} / \mathrm{mL}$. Compared to previously reported methods, the present assay method assessed extensive validation parameters as per $\mathrm{ICH}$ guidelines. The method has shown high precision, accuracy and adequate sensitivity and demands to be in use for further studies. The established method satisfies the system suitability criteria, peak integrity, and resolution of the drug peak. The overall results clearly indicate that the current method is attractive due to the good selectivity for quantitative determination of RMP in self-emulsifying lipid-based formulation and pharmaceutical formulations

\section{ACKNOWLEDGEMENT}

The authors would like to acknowledge Kayyali Chair for Pharmaceutical Industries for funding this project (KM-2017, Research Chair, Deanship of Scientific Research).

\section{REFERENCES}

1. Rao, K.S.S., K., RP-HPLC Method for the Determination of Losartan Potassium and Ramipril in Combined Dosage Form. Indian J Pharm Sci, 2010. 72(1), 108-11.

2. Rahman, N., Y. Ahmad, and S.N. Azmi,
Kinetic spectrophotometric method for the determination of ramipril in pharmaceutical formulations. AAPS Pharm Sci Tech, 2005. 6(3), 543-51.

3. Bhushan, R., D. Gupta, and S.K. Singh, Liquid 
chromatographic separation and UV determination of certain antihypertensive agents. Biomed Chromatogr, 2006, 20(2). 217-24.

4. Singh, M.K.P., D. Kulhari, H. Jain, S. K. Sistla, R. Chauhan, A. S., Poly (amidoamine) dendrimer-mediated hybrid formulation for combination therapy of ramipril and hydrochlorothiazide. Eur J Pharm Sci, 2017. 96, 84-92.

5. Kurade, V.P., M.G. Pai, and R. Gude, RPHPLC Estimation of Ramipril and Telmisartan in Tablets. Indian J Pharm Sci, 2009. 71(2): p. 148-51.

6. Shafiq, S.S., F. Talegaonkar, S. Ahmad, F. J. Khar, R. K. Ali, M., Development and bioavailability assessment of ramipril nanoemulsion formulation. Eur J Pharm Biopharm, 2007, 66(2), 227-43.

7. Shafiq, S., F. Shakeel, and R.K. Khar, Enhanced stability of ramipril in nanoemulsion containing cremophor-EL: a technical note. AAPS Pharm Sci Tech, 2008. 9(4), 1097-101.

8. M. De Diego, G.G., S. Mennickent, M. Olivares, R. Godoy. , Stress degradation studies of ramipril by a validated stabilityindicating liquid chromatographic method. J. Chil. Chem. Soc., 2010, 55(4).

9. Sankar, A.S., T. Vetrichelvan, and D. Venkappaya, Simultaneous estimation of ramipril, acetylsalicylic acid and atorvastatin calcium by chemometrics assisted UVspectrophotometric method in capsules. Acta
Pharm, 2011. 61(3), 283-96.

10. Abd el, H.S., Colyer, C. L., Hassan, W. S., Shalaby, A., Utility of 4-chloro-7nitrobenzofurazan (NBD-Cl) for the Spectrophotometric and spectrofluorometric determination of several antihistamine and antihypertensive drugs. J AOAC Int, 2013, 96(5),968-75.

11. Abdellatef, H.E., Spectrophotometric and spectrofluorimetric methods for the determination of ramipril in its pure and dosage form. Spectrochim Acta A Mol Biomol Spectrosc, 2007, 66(3), 701-6.

12. US Dept. of Health and Human Services, F.D.A., Centre for Drug Evaluation and Research (CDER), Centre for Veterinary Medicine (CVM), Guidance for Industry, in Bioanalytical Method Validation Rockville, MD, USA. 2001.

13. Mohsin, K., S. Al-Qahtani, and F.K. Alanazi, Rapid and sensitive bioanalytical stabilityindicating method for quantification of talinolol, a selective beta1 adrenoceptor antagonist in lipid based formulations using ultrafast UHPLC systems. Acta Pol Pharm, 2014, 71(5), 737-45.

14. Mansoor Alghazi, F.A., Kazi Mohsin, Nasir Ali Siddiqui, Faiyaz Shakeel, Nazrul Haq, Simultaneous separation of antihyperlipidemic drugs by green ultrahighperformance liquid chromatographyediode array detector method: Improving the health of liquid chromatography. Journal of Food and Drug Analysis, 2017, 25, 430-437. 